\title{
Magnetocrystalline anisotropy of FePt: A detailed view
}

\author{
Saleem Ayaz Khan, ${ }^{1}$ Peter Blaha, ${ }^{2}$ Hubert Ebert, ${ }^{3}$ Jan Minár ${ }^{1,3}$ and Ondřej Šipr ${ }^{1,4}$ \\ ${ }^{1}$ New Technologies Research Centre, University of West Bohemia, Univerzitní 2732, 30614 Pilsen, Czech Republic \\ ${ }^{2}$ Institute of Materials Chemistry, TU Vienna, Getreidemarkt 9, A-1060 Vienna, Austria \\ ${ }^{3}$ Universität München, Department Chemie, Butenandtstr. 5-13, D-81377 München, Germany \\ ${ }^{4}$ Institute of Physics ASCR v. v. i., Cukrovarnická 10, CZ-162 53 Prague, Czech Republic
}

(Received 1 April 2016; revised manuscript received 10 August 2016; published 24 October 2016)

\begin{abstract}
To get a reliable $a b$ initio value for the magnetocrystalline anisotropy (MCA) energy of FePt, we employ the full-potential linearized augmented plane wave (FLAPW) method and the full-potential Korringa-Kohn-Rostoker (KKR) Green function method. The MCA energies calculated by both methods are in good agreement with each other. As the calculated MCA energy significantly differs from experiment, it is clear that many-body effects beyond the local density approximation are essential. It is not really important whether relativistic effects for FePt are accounted for by solving the full Dirac equation or whether the spin-orbit coupling (SOC) is treated as a correction to the scalar-relativistic Hamiltonian. From the analysis of the dependence of the MCA energy on the magnetization angle and on the SOC strength it follows that the main mechanism of MCA in FePt can be described within second order perturbation theory. However, a distinct contribution not accountable for by second order perturbation theory is present as well.
\end{abstract}

DOI: 10.1103/PhysRevB.94.144436

\section{INTRODUCTION}

The various $a b$ initio electronic structure codes use different approaches to solve the Schrödinger equation for a solid. Usually different codes and/or methods yield results that are similar but show sometimes important differences in the details. These details start to matter if one aims at highprecision calculations with predictive power. Therefore an effort has lately intensified to standardize ab initio calculations and to find the conditions that have to be met so that reliable "true" quantitative values are obtained. So far the attention has been paid mostly to total energies, equilibrium lattice parameters, and bulk moduli [1-4]. We want to extend this effort to another numerically sensitive area, namely, to the magnetocrystalline anisotropy (MCA).

The MCA is manifested by the fact that the energy of a magnetically ordered material depends on the direction of the magnetization $\boldsymbol{M}$ with respect to the crystal lattice. It is an interesting phenomenon both for fundamental and technological reasons, as the MCA is important among others for the design of magnetic recording media. Theoretical research on MCA proceeds in two directions. First, one tries to understand the mechanism behind the MCA in simple intuitive terms, so that one would have guidance in search for materials with a high MCA energy [5-7]. Second, one tries to find which computational procedures have to be employed so that one can make quantitative predictions on the MCA energy [8-10].

Getting an accurate value of the MCA energy $E_{\mathrm{MCA}}$ is quite difficult as one has to, at least in principle, subtract two very large numbers (total energies for two orientations of magnetization) to get a very small number, namely $E_{\mathrm{MCA}}$. Several conditions for getting accurate well-converged results were explored in the past. In particular, the importance of a sufficiently dense mesh in the Brillouin zone (BZ) for the $\boldsymbol{k}$ space integration was recognized [11-13]. When dealing with supported systems such as adatoms or monolayers, the semiinfinite substrate has to be properly accounted for $[14,15]$. Despite all the efforts, getting accurate and reliable predictions of the MCA energy is still a problem. Numerical uncertainties severely restrict the practical usefulness of calculations of the MCA. They hinder our understanding of the underlying physics as well, because lack of reliable numerical values means that it is not really possible to determine which physical approximations and models are acceptable and which are not.

In this paper we focus on MCA of bulk FePt. This compound has the largest MCA energy of all bulk materials formed by transition metals and its crystal structure is quite simple, so it is a good candidate for a reliable calculation. At the same time, the presence of $\mathrm{Pt}$ - a heavy element—suggests that relativistic effects should be significant, offering thus an interesting possibility to check how different methods of dealing with relativistic effects, in particular with the spin-orbit coupling, influence the results. Besides, a deeper understanding of the MCA of FePt is important regarding the current search for suitable rare-earth-free magnetic materials. Transition metals are natural candidates in this respect and attracted a lot of attention recently $[16,17]$.

Previous theoretical studies on FePt based on the local density approximation (LDA) give a large spread of resultsfrom $1.8 \mathrm{meV}$ to $4.3 \mathrm{meV}[9,12,18-24]$. If one restricts to full potential methods only, one still gets a relatively large difference between various studies: $E_{\mathrm{MCA}}$ of $\mathrm{FePt}$ was determined as $2.7 \mathrm{meV}$ by FP-LMTO calculation of Ravindran et al. [12] and FLAPW calculation of Shick and Mryasov [23], $3.1 \mathrm{meV}$ by plane-waves calculation of Kosugi et al. [25], and $3.9 \mathrm{meV}$ by FP-LMTO calculation of Galanakis et al. [22]. The differences between various LDA calculations are comparable to the differences between LDA results and the experimental value of $1.3 \mathrm{meV}$ [26]. Even though part of the spread of the LDA results can be attributed to the use of different LDA exchange-correlation functionals, the differences are still too large to be acceptable. Besides, they occur also for studies which use the same exchange-correlation functional (e.g., both Ravindran et al. [12] and Galanakis et al. [22] use von Barth and Hedin functional [27]). This suggests that the accuracy of 
ab initio MCA energy calculations may not even be sufficient to answer the fundamental question whether the LDA itself is able to reproduce the experimental MCA energy of FePt or not.

Deciding which method gives better MCA results than the other is quite difficult, among other reasons because different computational approaches used by different codes are intertwined with different ways of implementing relativistic effects. Recall that as the MCA is intimately related to the spin orbit coupling (SOC), the way the relativity is included can be an important factor. To verify that a calculated MCA energy really represents the true LDA value, one has to use two different methods and make sure that the calculations are properly converged.

The aim of our work is to perform a robust and reliable LDA calculation of the MCA energy of FePt to find out whether treating relativistic effects via an explicit SOC Hamiltonian is sufficient for MCA calculations, whether the MCA of FePt can be described within the LDA, and what is the accuracy of current MCA energy calculations in general.

The first computational method we employ is the wellestablished and recognized full potential linearized augmented plane wave (FLAPW) method as implemented in the WIEN2K code [28]. This method was used as a reference in the recent study of the accuracy of total energies and related quantities [2-4]. Relativistic effects are treated approximately in WIEN2K, by introducing a separate SOC-related term to the Hamiltonian. As the second method we opted for a fully relativistic full potential multiple scattering KKR (Korringa-Kohn-Rostoker) Green function formalism as implemented in the SPRKKR code $[29,30]$.

Many aspects of the MCA of FePt were theoretically investigated in the past already. Daalderop et al. [18] and Ravindran et al. [12] studied the influence of the band filling on $E_{\mathrm{MCA}}$ of FePt. Many groups studied the influence of the temperature on the MCA of FePt [31,32]. The dependence of the Curie temperature on the FePt grain size was investigated via model Hamiltonian calculations [33]. Burkert et al. [9], Lukashev et al. [34], and Kosugi et al. [25] studied how $E_{\mathrm{MCA}}$ depends on the strain (i.e., the $c / a$ ratio). Cuadrado et al. [35] gradually substituted the $\mathrm{Fe}$ atom by $\mathrm{Cr}, \mathrm{Mn}, \mathrm{Co}, \mathrm{Ni}$, or $\mathrm{Cu}$ to find that the MCA energy of $\mathrm{Fe}_{1-y} \mathrm{X}_{y} \mathrm{Pt}$ alloys can be tuned by adjusting the content of the substituting element.

To facilitate the understanding of the MCA of FePt and related systems further, we focus on some aspects that have not been paid attention to so far. We show that if WIEN2K and SPRKKR calculations are converged, they yield comparable values for the MCA energy. Dealing with relativity by introducing an additional SOC-related term to the Hamiltonian is thereby justified. The theoretical MCA energy of FePt $(3.0 \mathrm{meV})$ is significantly larger than the experimental value (1.3 meV), implying conclusively that the LDA cannot properly describe the MCA of FePt. We also analyze how the total energy varies with the magnetization angle and how MCA energy scales with the SOC strength. Based on this we conclude that even though the MCA of FePt is dominated by a second order perturbation theory mechanism, there is a small but distinct contribution originating from the Pt sites which is not accountable for by second order perturbation theory.

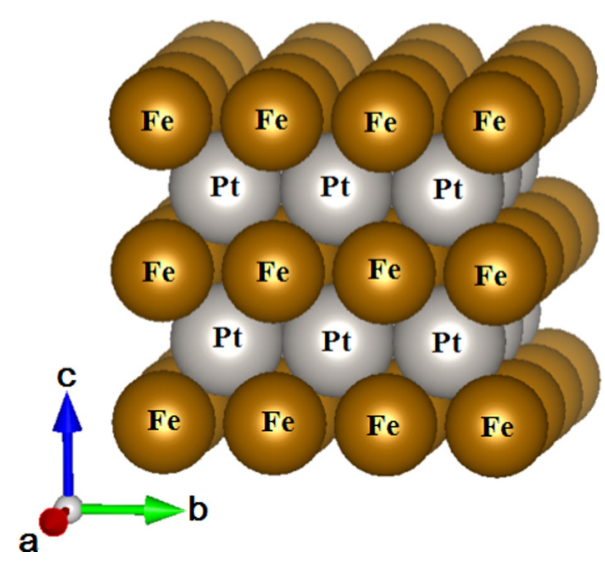

FIG. 1. Crystal structure of bulk $\mathrm{L} 1_{0} \mathrm{FePt}$.

\section{COMPUTATIONAL DETAILS}

\section{A. Technical details}

We studied bulk FePt with the $\mathrm{L} 1_{0}$ layered structure. The lattice parameters of a $t P 2$ unit cell are $a=2.722 \AA$ and $c=3.714 \AA$. Fe atoms and $\mathrm{Pt}$ atoms are at the $(0.0,0.0,0.0)$ and $(0.5,0.5,0.5)$ crystallographic positions, respectively, resulting in a compound with alternating $\mathrm{Fe}$ and $\mathrm{Pt}$ atomic layers stacked along the $c$ axis (see Fig. 1).

We used two different computational methods, namely, the FLAPW method as implemented in the WIEN2K code [28] and the multiple scattering KKR Green function method as implemented in the SPRKKR code $[29,30]$. Our calculations are based on the LDA. The values presented in the Results section (Sec. III) were obtained using the Vosko, Wilk, and Nusair (VWN) exchange-correlation functional [36]. Use of different LDA functionals leads to small but identifiable changes in $E_{\mathrm{MCA}}$, as explored in Sec. III E.

The KKR Green function calculations were done in the full-potential mode. Only when studying the scaling of $E_{\mathrm{MCA}}$ with the SOC strength (Sec. IIID), we rely on the atomic spheres approximation (ASA), because in that case many evaluations of $E_{\mathrm{MCA}}$ have to be done and the focus in that part is on trends and not so much on numerical values. The energy integrals were evaluated by contour integration on a semicircular path within the complex energy plane, using a Gaussian mesh of 40 points. An important convergence parameter is the maximum angular momentum $\ell_{\max }^{(\mathrm{KKR})}$ used for the multipole expansion of the Green function (see the Supplemental Material [37]). To get as accurate results as possible, we mostly use $\ell_{\max }^{(\mathrm{KKR})}=7$. However, if a lot of calculations with different settings have to be done (Secs. III C and IIID), we use $\ell_{\max }^{(\mathrm{KKR})}=3$, which is sufficient if the focus is on how $E_{\mathrm{MCA}}$ varies with the magnetization angle or with the SOC strength and not on particular values.

The convergence of FLAPW calculations is determined by the size of the basis. We treated $\mathrm{Fe} 3 p, 3 d, 4 s$ and $\mathrm{Pt}$ $5 p, 5 d, 6 s$ states as valence states and $\mathrm{Fe} 1 s, 2 s, 2 p, 3 s$ and $\mathrm{Pt}$ $1 s, 2 s, 2 p, 3 s, 3 p, 3 d, 4 s, 4 p, 4 d, 4 f, 5 s$ states as core states. The expansion of the wave functions into plane waves is controlled by the plane wave cutoff in the interstitial region. This cutoff is specified via the product $R_{\mathrm{MT}} K_{\mathrm{max}}$, where $R_{\mathrm{MT}}$ is the smallest muffin-tin ("atomic") sphere radius and $K_{\max }$ is the magnitude 
of the largest wave vector. We use $R_{\mathrm{MT}} K_{\max }=8$ in this study. The convergence of $E_{\mathrm{MCA}}$ with $R_{\mathrm{MT}} K_{\max }$ is investigated in the Supplemental Material [37]. The expansion of the wave functions into atomiclike functions inside the spheres is controlled by the angular-momentum cutoff $\ell_{\max }^{(\mathrm{APW})}$. We use $\ell_{\max }^{(\mathrm{APW})}=10$ throughout this paper. Note that the cutoffs $\ell_{\max }^{(\mathrm{APW})}$ and $\ell_{\max }^{(\mathrm{KKR})}$ have different roles in FLAPW and KKR-Green function methods, so their values cannot be directly compared.

As concerns the muffin-tin radii in WIEN2K calculations, the atomic spheres are chosen so that they are smaller than the touching spheres for the MCA energy calculations $\left(R_{\mathrm{MT}}^{(\mathrm{Fe})}=2.2\right.$ a.u., $R_{\mathrm{MT}}^{(\mathrm{Pt})}=2.3$ a.u., $R_{\mathrm{MT}}^{\text {(touch) }}=2.527$ a.u.), because in this way the basis avoids the linearization error. On the other hand, for analyzing site-related magnetic moments we use touching muffin-tin spheres because in this way we minimize the moments associated with the interstitial region. In this way we are in a better position to compare the WIEN2K results with the SPRKKR data, where the site-related magnetic moments are determined as moments within Voronoi polyhedra. The stability of $E_{\mathrm{MCA}}$ with respect to $R_{\mathrm{MT}}$ 's variation is demonstrated in the Supplemental Material [37].

Once the Green function components or the wave functions have been determined, the charge density is obtained via the $\boldsymbol{k}$-space integration over the BZ. When using the WIEN2K code, the BZ integration was carried out using the modified tetrahedron method [38]. When using the SPRKKR code, the BZ integration was carried out via sampling on a regular $\boldsymbol{k}$ mesh, making use of the symmetry [39]. The convergence of $E_{\mathrm{MCA}}$ with respect to the the number of $\boldsymbol{k}$ points is explored in the Supplemental Material [37]. Based on it, we used 800000 $\boldsymbol{k}$ points in the full BZ for WIEN2K calculations and $100000 \boldsymbol{k}$ points in the full BZ for SPRKKR calculations. Considering the convergence tests as a whole, we argue that that the numerical accuracy of our $E_{\mathrm{MCA}}$ values is about $0.1 \mathrm{meV}$ for WIEN2K calculations and about $0.2 \mathrm{meV}$ for SPRKKR calculations.

\section{B. Treatment of relativistic effects}

The SPRKKR code works fully relativistically; it solves a four-component Dirac equation by default. SOC is therefore implicitly fully included for all states. Nevertheless, the bare effect of the SOC can be investigated via SPRKKR if one employs an approximate two-component scheme [40] where the SOC-related term is identified by relying on a set of approximate radial Dirac equations. This scheme was used recently to investigate how the MCA energy of adatoms and monolayers on noble metals varies if SOC is selectively switched on only at some sites [15]. We employed it here for the same purpose.

As concerns the WIEN2K code, SOC is included differently for core and valence electrons. The core electrons are treated fully relativistically by solving the atomiclike Dirac equation. For the valence electrons the SOC is included in atomic spheres via an approximative scheme that introduces an additional term

$$
H_{\mathrm{SOC}}=\xi(\boldsymbol{r}) \boldsymbol{L} \cdot \boldsymbol{S}
$$

to the spin-polarized Schrödinger-like scalar relativistic equation. Technically, the influence of the term (1) is included by starting with a scalar-relativistic FLAPW calculation without SOC. The eigenfunctions thus obtained are then used as a basis in which another diagonalization is done, and this time the SOC term Eq. (1) is also taken into account. This procedure is often called second variational step [41]. Usually this second variational step is applied only to a subset of FLAPW eigenstates to gain a substantial speed-up. This subset is defined so that it includes all scalar-relativistic eigenstates up to energy $E_{\max }$ above the Fermi level. The $E_{\max }$ parameter thus plays an analogous role as $R_{\mathrm{MT}} K_{\max }$. Moreover, relativistic local orbitals ( $p_{1 / 2}$ wave functions) were added to the basis [42]. To achieve the highest accuracy, we set $E_{\max }$ as large as needed to include all FLAPW eigenfunctions in the second step (this can achieved by setting $E_{\max }$ of 100 Ry or higher). More details can be found in the Supplemental Material [37]. As concerns the interstitial region, valence electrons are treated in a nonrelativistic way. In the rest of this paper "fully relativistic calculation" implies use of the Dirac equation for SPRKKR and Schrödinger equation plus separate SOC term (1) in the Hamiltonian for WIEN2K, unless it is explicitly said otherwise.

The SPRKKR and WIEN2K codes allow for nonrelativistic and scalar-relativistic calculations as well. In the first case, both valence and core electrons are treated nonrelativistically. In the second case, the valence electrons are treated using the scalar-relativistic approach while for the core electrons atomistic Dirac equation is solved (this applies to both codes).

\section{Scaling of the spin-orbit coupling}

For a deeper understanding we want to investigate how $E_{\mathrm{MCA}}$ depends on the SOC. More specifically, we are interested in how $E_{\mathrm{MCA}}$ varies if the SOC strength is varied at the Fe and Pt sites separately, i.e., we assume that the Hamiltonian Eq. (1) can be symbolically rewritten as

$$
H_{\mathrm{SOC}}=\sum_{i} \lambda_{i} \xi_{i}(\boldsymbol{r}) \boldsymbol{L}_{i} \cdot \boldsymbol{S}_{i}
$$

where $\lambda_{i}$ is the scaling factor for site $i$. Such calculations were done via the SPRKKR code, using the approximate scheme [40] mentioned in the beginning of Sec. II B.

\section{RESULTS}

\section{A. Magnetic moments}

The presence of Pt in FePt suggests that the way relativistic effects are treated could be important. Therefore, we calculated magnetic moments in FePt using a nonrelativistic Schrödinger equation, using a scalar-relativistic approach, and using a relativistic scheme. Spin magnetic moments related either to the unit cell or only to the Fe site are shown in Table I.

TABLE I. Spin magnetic moments (in $\mu_{B}$ ) related either to a FePt unit cell or just to the Fe site, for different ways of including the relativistic effects.

\begin{tabular}{lccccc}
\hline \hline & \multicolumn{2}{c}{ SPRKKR } & & \multicolumn{2}{c}{ WIEN2K } \\
\cline { 2 - 3 } \cline { 5 - 6 } & $\mu_{\text {spin }}^{(\text {cell) }}$ & $\mu_{\text {spin }}^{(\mathrm{Fe})}$ & & $\mu_{\text {spin }}^{(\text {(cell) }}$ & $\mu_{\text {spin }}^{(\mathrm{Fe})}$ \\
\hline non relativistic & 3.17 & 2.86 & 3.15 & 2.86 \\
scalar relativistic & 3.21 & 2.86 & 3.21 & 2.87 \\
fully relativistic & 3.17 & 2.83 & 3.17 & 2.84 \\
\hline \hline
\end{tabular}


TABLE II. Orbital magnetic moments (in $\mu_{B}$ ) related to the Fe and $\mathrm{Pt}$ atoms in $\mathrm{FePt}$ for magnetization either parallel to the $z$ axis $\left(\mu_{\mathrm{orb}}^{(\boldsymbol{M} \| z)}\right)$ or perpendicular to the $z$ axis $\left(\mu_{\mathrm{orb}}^{(\boldsymbol{M} \| x)}\right)$.

\begin{tabular}{lccccc}
\hline \hline & \multicolumn{3}{c}{ Fe } & & \multicolumn{2}{c}{ Pt } \\
\cline { 2 - 3 } \cline { 5 - 6 } & SPRKKR & WIEN2K & & SPRKKR & WIEN2K \\
\hline$\mu_{\text {orb }}^{(\boldsymbol{M} \| z)}$ & 0.065 & 0.065 & & 0.044 & 0.042 \\
$\mu_{\text {orb }}^{(\boldsymbol{M} \| x)}$ & 0.062 & 0.062 & & 0.060 & 0.054 \\
\hline \hline
\end{tabular}

We can see that relativity has only a marginal effect on the spin magnetic moments in FePt. Orbital magnetic moments are more interesting in this respect-they would be zero in the absence of SOC. Our results in Table II give the orbital magnetic moment at the Fe and Pt sites for two orientations of the magnetization.

One can see that both codes lead to very similar values for $\mu_{\text {spin }}$ and $\mu_{\text {orb }}$. In particular, the anisotropy of $\mu_{\text {orb }}$ at Fe and at Pt sites is nearly the same. Small differences between the codes in the local magnetic moments may be due to the fact that the moments are defined in different regions: Wigner-Seitz cells (or more precisely Voronoi polyhedra) in SPRKKR and touching muffin-tin spheres in WIEN2K. The difference would be larger if we used a "standard" setting of muffin-tin radii in WIEN2K $\left(R_{\mathrm{MT}}^{(\mathrm{Fe})}=2.2\right.$ a.u. and $R_{\mathrm{MT}}^{(\mathrm{Pt})}=2.3$ a.u. instead of $R_{\mathrm{MT}}^{(\mathrm{Fe})}=R_{\mathrm{MT}}^{(\mathrm{Pt})}=$ 2.527 a.u.): In that case, the local spin moments obtained via WIEN2K would be smaller by about $3 \%$ and orbital moments by about $10 \%$.

\section{B. Magnetocrystalline anisotropy energy}

Calculating the MCA energy by subtracting total energies for two orientations of the magnetization as

$$
E_{\mathrm{MCA}} \equiv E^{(\boldsymbol{M} \| x)}-E^{(\boldsymbol{M} \| z)}
$$

is very challenging, because the total energies and the MCA energy differ by about eight or nine orders of magnitude. We paid a lot of attention to the issues of convergence to get accurate numbers. The details can be found in the Supplemental Material [37]. Here we only mention two issues which have to be given special attention.

For full-potential SPRKKR calculations, attention has to be paid to the multipole expansion of the Green function governed by the cutoff $\ell_{\max }^{(\mathrm{KKR})}$. KKR calculations have known behavior concerning the $\ell_{\max }^{(\mathrm{KKR})}$ convergence which plays a role if one aims at high-accuracy total energy calculations [43,44]. Part of the problem is numerical difficulties connected with the evaluation of the Madelung contribution to the full potential for high angular momenta $[45,46]$. Note that to obtain the Green function components up to $\ell_{\max }^{(\mathrm{KKR})}$, one needs potential components up to $2 \ell_{\max }^{(\mathrm{KKR})}$ and shape functions components up to $4 \ell_{\max }^{(\mathrm{KKR})}$. Another difficulty is an efficient treatment of the socalled near-field corrections [45,47]. Various ways to deal with these issues have been suggested [44,47-49]. We performed a test of the $\ell_{\max }^{(\mathrm{KKR})}$ convergence (Supplemental Material [37]) which indicates that if the $\ell_{\max }^{(\mathrm{KKR})}=7$ cutoff is used, that the numerical accuracy of the MCA energy is about $0.2 \mathrm{meV}$.

For accurate MCA energy calculations using the WIEN2K code, one has to pay special attention so that the energy pa-
TABLE III. MCA energy $E_{\max }$ of FePt (in meV) calculated by two approaches.

\begin{tabular}{lcc}
\hline \hline & SPRKKR & WIEN2K \\
\hline subtracting total energies & 3.04 & 2.99 \\
magnetic force theorem & 3.12 & 2.85 \\
\hline \hline
\end{tabular}

rameters $E_{\ell}$ used for calculating radial wave functions $u_{\ell}\left(r, E_{\ell}\right)$ are determined very precisely and consistently. This applies, in particular, also for the relativistic local orbitals. In WIEN2K this is done by searching for the energies where $u_{\ell}\left(R_{M T}, E\right)$ changes the sign to determine $E_{\text {top }}$, and where it has zero slope to determine $E_{\text {bottom }}$. The arithmetic mean of these two energies gives $E_{\ell}$. For the calculations presented here these energies had to be determined with an accuracy better than $0.1 \mathrm{mRy}$. A parameter specific for relativistic calculations via WIEN2K is $E_{\max }$, which controls how many scalar-relativistic eigenstates are considered when SOC is included (Supplemental Material [37]). We used $E_{\max }=100 \mathrm{Ry}$, meaning that all eigenstates were included.

The MCA energy obtained by subtracting the total energies is shown in the first line of Table III. Values obtained via SPRKKR and WIEN2K show good agreement. Considering the convergence analysis we performed, this allows us to state that the magnetic easy axis of FePt is out-of-plane and the MCA energy is $3.0 \mathrm{meV}$ within the LDA framework (for the VWN exchange-correlation functional).

Obtaining the MCA energy by subtracting the total energies is computationally very costly. The need for self-consistent calculations for two magnetization directions can be avoided if one relies on the magnetic force theorem. In this approach the MCA energy is calculated using a frozen spin-dependent potential [50,51]. The MCA energy is then obtained either by subtracting the band energies or by evaluating the torque at magnetization tilt angle of $45^{\circ}[15,52]$. As the magnetic force theorem is frequently employed, we applied it here as well. The results are shown in the second line of Table III. We can see that the magnetic force theorem yields very similar values as if total energies are subtracted.

\section{Relation between $E_{\mathrm{MCA}}$ and anisotropy of $\mu_{\mathrm{orb}}$}

For the sake of completeness we checked also the Bruno formula [53], which links the MCA energy to the anisotropy of orbital magnetic moment. The Bruno formula [53] (as well as the slightly more sophisticated van der Laan formula [5]) can be derived from second order perturbation theory if some additional assumptions are made. It is often employed in the context of x-ray magnetic circular dichroism experiments that give access to the anisotropy of orbital magnetic moment via the so-called sum rules.

Even though the formula was originally derived for systems with only one atomic type, the relation between the MCA energy and the anisotropy of orbital magnetic moments has been frequently applied also for multicomponent systems [12,54-58]. In such a case an estimate of $E_{\mathrm{MCA}}$ can be made by evaluating (cf. Ravindran et al. [12] and Andersson et al. 
[59])

$$
E_{\mathrm{MCA}}=\sum_{i} \frac{\xi_{i}}{4}\left(\mu_{\mathrm{orb}}^{(i, \boldsymbol{M} \| z)}-\mu_{\mathrm{orb}}^{(i, \boldsymbol{M} \| x)}\right),
$$

where $i$ labels the constituting atoms. This equation is valid only if off-site spin-flip terms are neglected [12,59,60].

We evaluated Eq. (4) using SOC parameters $\xi^{(\mathrm{Fe})}=65 \mathrm{meV}$ and $\xi^{(\mathrm{Pt})}=712 \mathrm{meV}$, as obtained from ab initio calculations for FePt relying on the method described by Davenport et al. [61]. We obtained $E_{\mathrm{MCA}}=-2.62 \mathrm{meV}$ using SPRKKR results and $E_{\mathrm{MCA}}=-2.09 \mathrm{meV}$ using WIEN2K results. The sign of $E_{\mathrm{MCA}}$ evaluated from Eq. (4) is wrong, indicating that this formula does not provide a suitable framework for studying the MCA of FePt. Technically, the reversal of the sign of $E_{\mathrm{MCA}}$ obtained via Eq. (4) is due to $\mu_{\text {orb }}$ at $\mathrm{Pt}$ (see Table II): We have $\mu_{\mathrm{orb}}^{(\boldsymbol{M} \| z)}>\mu_{\mathrm{orb}}^{(\boldsymbol{M} \| x)}$ at the Fe site and $\mu_{\mathrm{orb}}^{(\boldsymbol{M} \| x)}>\mu_{\mathrm{orb}}^{(\boldsymbol{M} \| z)}$ at the Pt site. As $\xi^{(\mathrm{Pt})}$ is much larger than $\xi^{(\mathrm{Fe})}$, the Pt-related term dominates in Eq. (4).

The failure of the Bruno formula (4) does not automatically imply that second order perturbation theory cannot be used for describing the MCA of FePt. Namely, it is likely that additional assumptions employed in the derivation of Eq. (4) are not fulfilled; in particular, for Pt atoms, the exchange splitting and SOC will be of the same order of magnitude. Two more indicative tests whether second order perturbation theory itself provides a good framework for understanding the MCA of FePt are presented below.

\section{Dependence of the total energy on the orientation of the magnetization axis}

Accurate calculations can provide information on the full form of the dependence of the total energy on the angle $\theta$ between the magnetization direction and the $z$ axis. For tetragonal systems the first two terms in the directional cosines expansion of the total energy are

$$
E(\theta)-E_{0}=K_{1} \sin ^{2} \theta+K_{2} \sin ^{4} \theta .
$$

Here we omit the azimuthal dependence, keeping $\phi=0^{\circ}$. If the influence of SOC is included via the explicit term Eq. (1), then application of second order perturbation theory leads to a simple dependence of the total energy on the angle $\theta$ as

$$
E(\theta)-E_{0}=K_{1} \sin ^{2} \theta,
$$

meaning that only the first term survives in Eq. (5) [53,62]. Inspecting the full $E(\theta)$ dependence as obtained via fullyrelativistic ab initio calculations thus provides the possibility to estimate to what degree a treatment of MCA based on second order perturbation theory is adequate: Large $K_{2}$ coefficient implies large deviations from second order perturbation theory.

We performed a series of calculations for different magnetization tilt angle $\theta$, using the SPRKKR code. The MCA energy was evaluated as a difference of total energies. The results are shown via circles in Fig. 2. Because we wanted to have a fine $\theta$ mesh, we had to perform a lot of calculations; therefore, we used $\ell_{\max }^{(\mathrm{KKR})}=3$ in this section. The numerical value for $\theta=90^{\circ}$ thus differs a bit from Table III, where the $\ell_{\max }^{(\mathrm{KKR})}=7$ cutoff was used.

The $a b$ initio data were fitted via Eq. (5). If only the $K_{1} \sin ^{2} \theta$ term is employed (taking $K_{2}=0$ ), we obtain $K_{1}=$
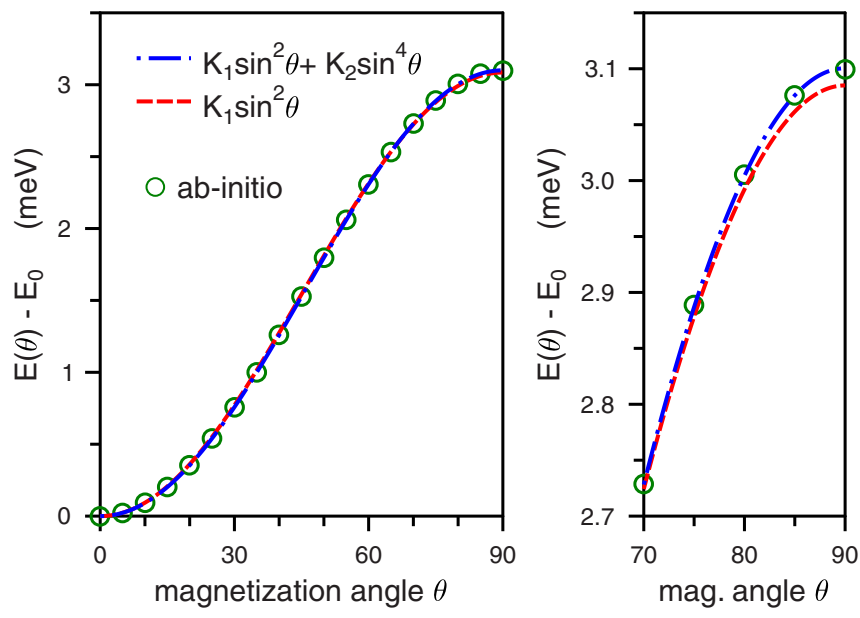

FIG. 2. Dependence of the total energy on the magnetization angle $\theta$ (circles) and its fit either as $K_{1} \sin ^{2} \theta$ (dashed line) or as $K_{1} \sin ^{2} \theta+K_{2} \sin ^{4} \theta$ (dash-dotted line). An overall view is in the left panel; a detailed view of the region close to $\theta=90^{\circ}$ is in the right panel.

$3.085 \mathrm{meV}$. If both terms in Eq. (5) are employed, we obtain $K_{1}=3.008 \mathrm{meV}$ and $K_{2}=0.092 \mathrm{meV}$. Even though both fits look nearly the same in the overall view, a detailed analysis shows that the fit with both terms is significantly better (cf. the right panel in Fig. 2). Using even higher order terms in the fit did not lead to a significant improvement.

To summarize, our calculations show that the dependence of the total energy on the magnetization angle is fully described by Eq. (5). The ratio of the coefficients $K_{2} / K_{1}$ is 0.03 , thus we deduce that the MCA of FePt is dominated by the second order perturbation theory, but there is also a small but identifiable contribution which cannot be described by it.

\section{Dependence of the MCA energy on spin orbit coupling}

If the magnetocrystalline anisotropy is described within second order perturbation theory, it scales with the square of the SOC-scaling parameter $\lambda, E_{\mathrm{MCA}} \sim \lambda^{2}[5,53,62]$. Inspecting the $E_{\mathrm{MCA}}(\lambda)$ dependence thus provides another criterion to what degree second order perturbation theory is sufficient to describe magnetocrystalline anisotropy of FePt. To get typespecific information, one should scale $\lambda_{\mathrm{Fe}}$ and $\lambda_{\mathrm{Pt}}$ separately. In that case, however, the scaling of $E_{\mathrm{MCA}}$ with SOC takes a somewhat more complicated form [59]

$$
E_{\mathrm{MCA}}\left(\lambda_{\mathrm{Fe}}, \lambda_{\mathrm{Pt}}\right)=A \lambda_{\mathrm{Fe}}^{2}+B \lambda_{\mathrm{Fe}} \lambda_{\mathrm{Pt}}+C \lambda_{\mathrm{Pt}}^{2} .
$$

The scaling of $E_{\mathrm{MCA}}$ with SOC will thus retain a quadratic form only if the scaling is uniform $\left(\lambda_{\mathrm{Fe}}=\lambda_{\mathrm{Pt}}\right)$ or if SOC for one of the atomic types is zero (recovering thus the case of a single-component system $[5,53,62])$.

We start by calculating $E_{\mathrm{MCA}}$ for a uniform SOC scaling, i.e., $\lambda_{\mathrm{Fe}}=\lambda_{\mathrm{Pt}}$. We vary $\lambda$ from 0 to 1.5 to cover the nonrelativistic as well as the relativistic regime: If $\lambda$ is zero, there is no spin orbit coupling; if $\lambda$ is 1 , we recover the standard relativistic case. The calculations were done with the SPRKKR code, employing the scheme described in Sec. II C and evaluating $E_{\mathrm{MCA}}$ by subtracting total energies. To reduce the computer requirements, we performed all the calculations 


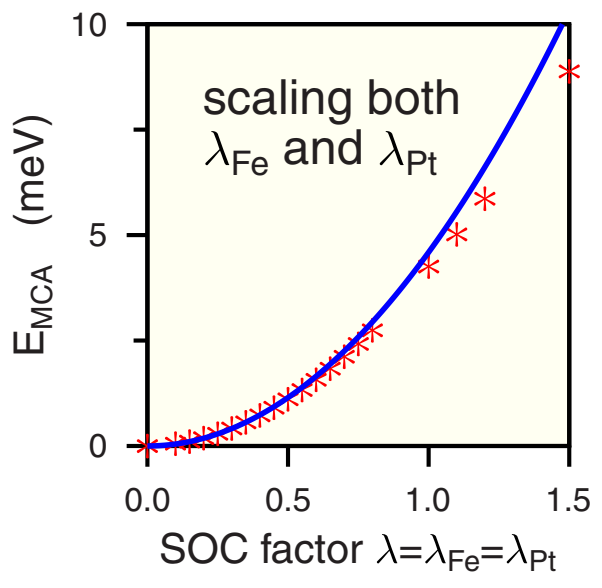

FIG. 3. Dependence of $E_{\mathrm{MCA}}$ on the SOC scaling factor $\lambda$. The markers denote calculated values of $E_{\mathrm{MCA}}$; the line represents a fit to these data within the $\lambda \in[0 ; 0.4]$ interval.

in this section with $\ell_{\max }^{(\mathrm{KKR})}=3$ in the ASA mode; this enables us to use a fine $\lambda$ mesh so that the curve fitting is reliable. The results are shown by points in Fig. 3. Employment of the ASA obviously leads to less accurate results than for full-potential calculations: $E_{\mathrm{MCA}}$ obtained within the ASA is by about $1 \mathrm{eV}$ larger than $E_{\mathrm{MCA}}$ obtained for full potential. However, this does not affect our conclusions concerning the scaling of $E_{\mathrm{MCA}}$ with strength of the SOC.

To verify the predictions of the perturbation theory, we fit calculated $E_{\mathrm{MCA}}(\lambda)$ with the quadratic function,

$$
E_{\mathrm{MCA}}(\lambda)=a \lambda^{2} \text {. }
$$

Perturbation theory should work well for small values of $\lambda$ while it can be less appropriate for large values of $\lambda$. So the fit to the function (7) is performed in such a way that the $a$ coefficient is sought only for $\lambda$ in the range between zero and 0.4 (the upper value was arbitrarily chosen just for convenience). One can see from Fig. 3 that while the fit describes the $a b$ initio data very well within the $\lambda \in[0 ; 0.4]$ range, there are small but clear deviations for larger $\lambda$. This suggests that while second order perturbation theory accounts for the dominant mechanism of magnetocrystalline anisotropy of FePt, some effects beyond it are also present.

To learn more about atom-specific contributions to MCA, let us scale the SOC at the Fe and Pt sites separately. When varying $\lambda_{\mathrm{Fe}}$ or $\lambda_{\mathrm{Pt}}$ we further distinguish two cases-either the SOC at the remaining species is totally suppressed $(\lambda=0)$ or it is kept at its "normal" value $(\lambda=1)$. Results for scaling SOC at the Fe sites are shown in Fig. 4; results for scaling $\mathrm{SOC}$ at the Pt sites are shown in Fig. 5. Fits to the quadratic dependence of $E_{\mathrm{MCA}}$ on $\lambda_{\mathrm{Fe}}$ or on $\lambda_{\mathrm{Pt}}$ were done only in the case where SOC at the other site is suppressed. Namely, if $\lambda$ at the other atomic type is nonzero, the functional dependence is more complicated—see Eq. (6)—and fitting $E_{\mathrm{MCA}}(\lambda)$ with the simple Eq. (7) would not make sense. Similarly as in the case of the uniform scaling, the fits were attempted for $\lambda$ in the $[0 ; 0.4]$ interval.

Concerning the case when SOC is varied at the Fe sites, one can see that if $\lambda_{\mathrm{Pt}}=0$, the dependence of $E_{\mathrm{MCA}}$ on $\lambda_{\mathrm{Fe}}$ is perfectly accounted for by second order perturbation theory:
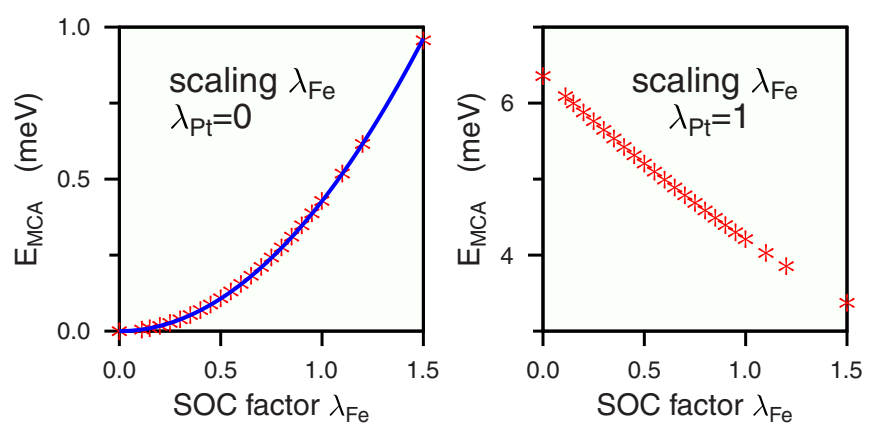

FIG. 4. Dependence of $E_{\mathrm{MCA}}$ on the SOC scaling factor at the Fe sites $\lambda_{\mathrm{Fe}}$. The markers denote calculated values of $E_{\mathrm{MCA}}$; the line in the left panel represents a fit to these data within the $\lambda_{\mathrm{Fe}} \in[0 ; 0.4]$ interval.

The quadratic fit describes the $E_{\mathrm{MCA}}\left(\lambda_{\mathrm{Fe}}\right)$ dependence very well also outside the [0;0.4] interval in which the $a$ coefficient was sought (left graph in Fig. 4). This suggests that it must be the strong SOC at Pt sites which makes the $E_{\mathrm{MCA}}(\lambda)$ curve in Fig. 3 to deviate from a perfect parabola. Indeed, if SOC at Pt sites is switched on (right graph in Fig. 4), the $E_{\mathrm{MCA}}(\lambda)$ functional dependence changes completely.

Let us turn now to the case of varying $\lambda_{\mathrm{Pt}}$. If there is no SOC at the Fe sites, the $E_{\mathrm{MCA}}\left(\lambda_{\mathrm{Pt}}\right)$ dependence is described by the fitted parabola only for low values of $\lambda_{\mathrm{Pt}}$ (left graph in Fig. 5). If $\lambda_{\mathrm{Pt}}$ increases beyond the fitting interval of [0;0.4], deviations of $a b$ initio data points from the fit by Eq. (7) are similar as for the uniform SOC fit presented in Fig. 3. So it follows from our analysis that the effect of SOC at the $\mathrm{Fe}$ sites can be accounted for by second order perturbation theory while the effect of SOC at the Pt sites goes beyond it.

\section{E. Dependence of the MCA energy on the LDA exchange-correlation functional}

Usually the calculated properties of solids do not crucially depend on which form of the LDA exchange-correlation functional is used. However, as the MCA energy is a very sensitive quantity, it is useful to investigate how the $E_{\mathrm{MCA}}$ varies if different LDA exchange-correlation functionals are used. Apart from the VWN exchange-correlation functional used throughout this work, we include in the comparison the
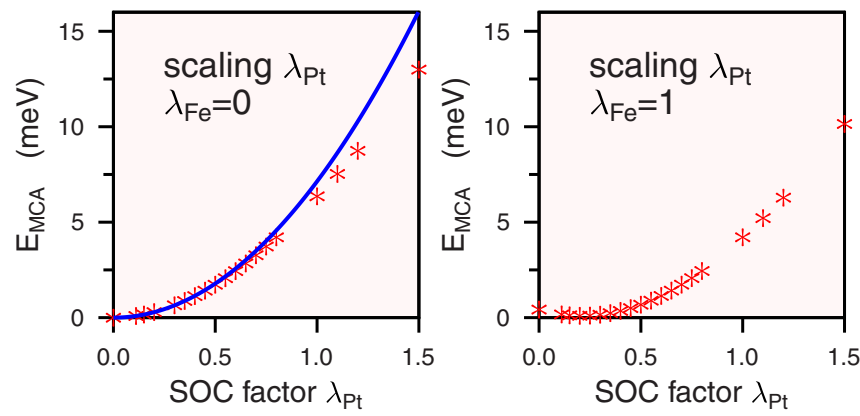

FIG. 5. Dependence of $E_{\mathrm{MCA}}$ on the SOC scaling factor at the $\mathrm{Pt}$ sites $\lambda_{\mathrm{Pt}}$. The markers denote calculated values of $E_{\mathrm{MCA}}$; the line in the left panel represents a fit to these data within the $\lambda_{\mathrm{Pt}} \in[0 ; 0.4]$ interval. 
TABLE IV. The MCA energy of FePt (in meV) calculated by subtracting total energies for different exchange and correlation functionals.

\begin{tabular}{lcc}
\hline \hline & SPRKKR & WIEN2K \\
\hline Vosko and Wilk and Nusair [36] & 3.04 & 2.99 \\
Perdew and Wang [63] & & 3.02 \\
von Barth and Hedin [27] & 3.29 & 3.18 \\
Moruzzi, Janak and Williams [64] & 2.97 & \\
\hline \hline
\end{tabular}

Perdew and Wang exchange-correlation functional [63] (the default for WIEN2K) and functionals suggested by von Barth and Hedin [27] and by Moruzzi, Janak, and Williams [64].

We evaluated $E_{\mathrm{MCA}}$ by subtracting total energies for this test. The results are summarized in Table IV. One can see that different LDA functionals lead to MCA energies that differ from each other by $0.1-0.2 \mathrm{meV}$.

\section{F. Relativistic effects in the density of states}

Figure 6 depicts the influence of relativity on the density of states (DOS) resolved in angular momentum components respective to $\mathrm{Fe}$ and $\mathrm{Pt}$ sites. The data presented here were
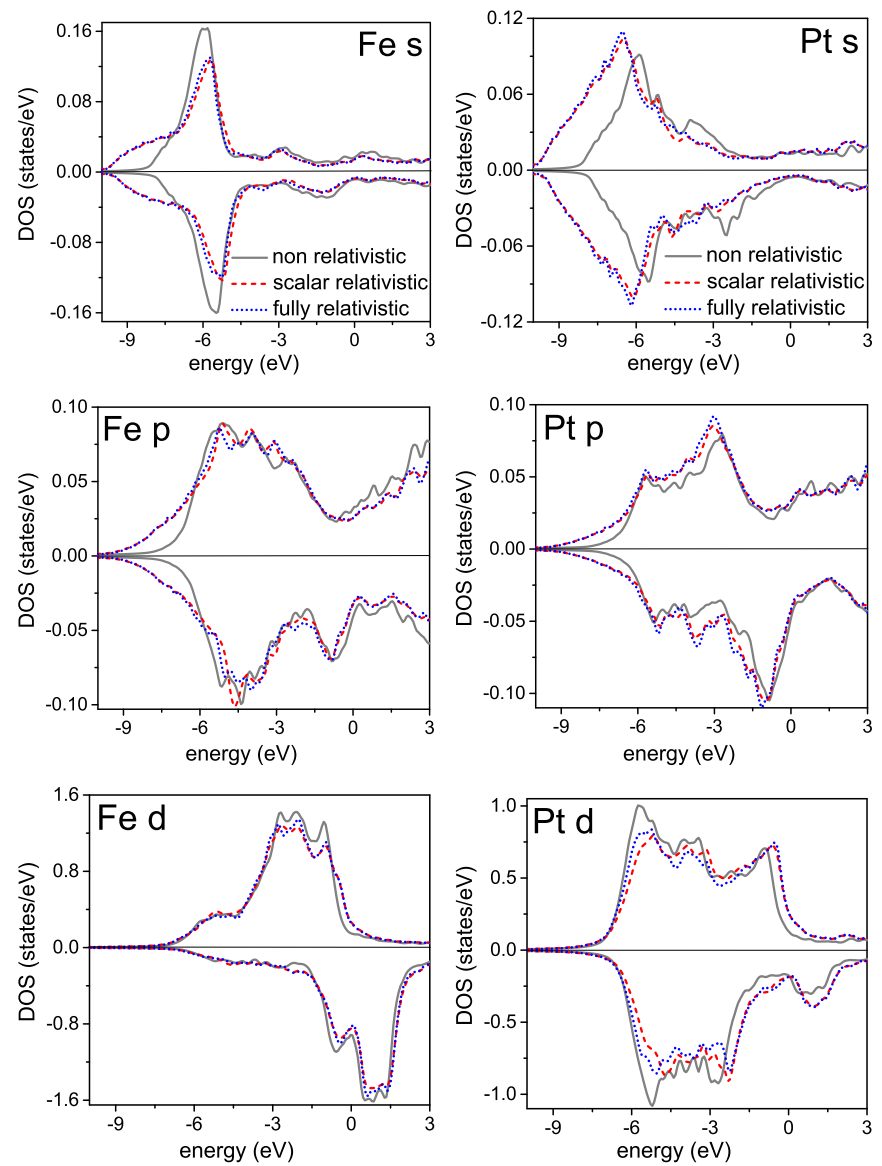

FIG. 6. Partial spin-resolved angular-momentum-projected density of states for $\mathrm{Fe}$ and $\mathrm{Pt}$ sites calculated within a nonrelativistic, a scalar-relativistic, and a fully-relativistic framework. obtained using the SPRKKR code; data obtained using the WIEN2K code look practically the same.

Generally, there is a significant change in the DOS when going from the nonrelativistic to scalar-relativistic case and only a minor change when going from the scalar-relativistic to the the fully relativistic case. The largest difference between the nonrelativistic and relativistic case is for the $s$ states. This may be due to the fact that $s$ electrons have a large probability density near the nucleus where relativistic effects (mass-velocity and Darwin term) are stronger than at larger distances. The largest difference between scalar relativistic and fully relativistic calculations is for the $\mathrm{Pt} d$ states, where the SOC is also expected to be stronger than for the other cases.

For $\mathrm{Pt} s$ and $d$ states one can make an interesting comparison with atomic results for $\mathrm{Au}$ [65] which are often quoted when relativistic effects in solids are discussed. It follows from Fig. 6 that relativistic effects shift valence Pt $6 s$ states to lower energies due to the orthogonality constrains to the more localized $1 s$ state and Pt $5 d$ states to higher energies due to a better screening of the nucleus by innermost electrons. The same happens for $6 s$ and $5 d$ atomic states of Au, respectively. So we can infer that the mechanism through which relativity affects Pt states is essentially atomiclike and common to all $5 d$ noble metals.

\section{DISCUSSION}

Our aim was to get reliable quantitative information on the MCA of FePt, which we take as an archetypal layered system of magnetic and nonmagnetic transition metals. We employed two quite different computational procedures. Both of them yield similar values for the MCA energy. Numerical stability of results is well documented by convergence tests presented in the Supplemental Material [37]. Therefore the results can be trusted to represent the true LDA value of the MCA energy. Our data can be used as a benchmark for LDA calculations.

Relativistic effects are implemented in the WIEN2K code in an approximative way, accounting for the SOC by a separate term [see Eq. (1)] which is added to the scalar-relativistic Hamiltonian. Most codes rely on this approach when they deal with SOC. The SPRKKR scheme, on the other hand, solves the Dirac equation so it does not use approximations when dealing with relativistic effects. Good agreement between MCA energies obtained via the WIEN2K code and via the SPRKKR code shows that dealing with relativity by invoking the separate term Eq. (1) is justified in our case. As we are studying FePt, i.e., a compound containing an element with a strong SOC, it is likely that the approximative scheme associated with Eq. (1) is sufficiently accurate for most common situations and/or systems. One should only make sure that a sufficiently large basis for the second variation step is taken (Supplementalry Material [37]).

We calculated $E_{\mathrm{MCA}}$ both via subtracting total energies and via the magnetic force theorem. Using the magnetic force theorem is technically much more convenient than subtracting total energies. Knowing limits of its reliability is thus vital. For pure $\mathrm{Fe}$ monolayers the magnetic force theorem was shown to be valid to a high accuracy $[66,67]$. However, there are indications that this may no longer be true for systems with normally nonmagnetic atoms with large induced moments 
and strong SOC $[10,68]$. For such atoms one would expect rather large changes of the spin-polarized electron density upon rotation of the magnetization. This applies also for the $\mathrm{Pt}$ atoms in FePt. Our results indicate, nevertheless, that the magnetic force theorem yields quite accurate values for $E_{\mathrm{MCA}}$ for FePt (Table III). One can conjecture that this would be the case for similar layered systems as well.

When comparing our $E_{\mathrm{MCA}}$ with experiment (1.3-1.4 meV) [26], it is evident that the LDA result does not quite agree with it. Clearly one has to go beyond LDA for a quantitative description of MCA of FePt. It does not matter in this respect which specific form of the LDA functional is used. Nevertheless, as different LDA functionals lead to similar but still visibly different values of $E_{\mathrm{MCA}}$ (cf. Table IV), each calculation of the MCA energy should be always accompanied by the information of which parametrization of the LDA functional was employed.

Employing the generalized gradient approximation (GGA) does not lead to substantial improvement with respect to the LDA. We obtained $E_{\mathrm{MCA}}=2.73 \mathrm{meV}$ for the frequently used PBE-GGA form [69] (using the WIEN2K code and evaluating the MCA energy as a difference of total energies). It is worth noting in this respect that Shick and Mryasov were able to obtain the MCA energy of FePt as $1.3 \mathrm{meV}$ by using the LDA $+U$ approach and searching for suitable site-related values of the $U$ parameter [23]. Interestingly, if many-body effects are described via the orbital polarization term of Brooks [70], calculated $E_{\mathrm{MCA}}$ is not significantly improved in comparison with the LDA [12,20,21,23]—-despite the fact that this approach proved to be useful when calculating orbital magnetic moments of transition metals [71,72].

The Bruno formula, derived originally for singlecomponent systems only, has recently been employed also for systems where there is more than one magnetic element $[12,54,55,57]$. In our case the Bruno formula suggests a wrong magnetic easy axis, hence it is not a suitable tool for understanding the MCA of FePt. Similar observations were made earlier for other compounds containing $3 d$ and $5 d$ elements [59,60,73], so we suggest that intuition based on analysis of orbital moments should not be used for these systems-despite its appeal and success in monoelemental systems.

Concerning a more detailed view on the mechanism of MCA, we found that even though MCA of FePt is dominated by a second order perturbation theory mechanism (as found earlier by Kosugi et al. [25] by analyzing the dependence of $E_{\mathrm{MCA}}$ of FePt on $c / a$ ), effects beyond it are clearly present as well. These effects could be identified (i) by analyzing the full angular dependence of the total energy and (ii) by inspecting how the MCA energy depends on the SOC strength. Separate scaling of SOC at Fe and Pt sites allows us to deduce that the deviations from a pure second order perturbation theory mechanism have their origin at the $\mathrm{Pt}$ sites. One possible mechanism that is beyond the standard second order perturbation theory is reoccupation of states close to the Fermi level $[7,18]$.

Another implication coming from our analysis of the full angular dependence of the total energy is that one can indeed use the torque implementation of the magnetic force theorem: Replacing the difference of energies $E\left(90^{\circ}\right)-E\left(0^{\circ}\right)$ by the torque at $45^{\circ}$ can be done only if Eq. (5) is valid [15,52]. It follows from the results shown in Fig. 2 that this indeed is the case.

\section{CONCLUSIONS}

If electronic structure calculations performed by means of FLAPW and KKR methods are properly converged, they yield the same results even for such sensitive quantities as the magnetocrystalline anisotropy energy. The proper LDA value of the MCA energy for FePt $(3.0 \mathrm{meV}$ for the VWN exchange-correlation functional) is significantly larger than in experiment $(1.3 \mathrm{meV})$, meaning that the MCA of FePt can be described properly only if many-body effects beyond the LDA are included. As our value of $E_{\mathrm{MCA}}$ was obtained by two different methods and the convergence of both of them was carefully checked, it can be used as a benchmark in future calculations.

It is not really important whether relativistic effects for FePt are accounted for by solving the full Dirac equation or whether the spin-orbit coupling is treated as a correction to the scalar-relativistic Hamiltonian. The main mechanism of MCA in FePt can be described within the framework of second order perturbation theory, but a significant contribution not accountable for by the second order perturbation theory is present as well.

\section{ACKNOWLEDGMENTS}

We would like to acknowledge CENTEM project (CZ.1.05/2.1.00/03.0088), CENTEM PLUS (LO1402), and COST CZ LD15147 of The Ministry of Education, Youth and Sports (Czech Republic). Computational time has been provided with the MetaCentrum (LM205) and CERITSC (CZ.1.05/3.2.00/08.0144) infrastructures. In addition we would like to thank the travel support from COST action MP1306 (EUSpec).
[1] M. Asato, A. Settels, T. Hoshino, T. Asada, S. Blügel, R. Zeller, and P. H. Dederichs, Phys. Rev. B 60, 5202 (1999).

[2] K. Lejaeghere, V. V. Speybroeck, G. V. Oost, and S. Cottenier, Rev. Solid State Mater. Sci. 39, 1 (2014).

[3] Comparing solid state DFT codes, basis sets and potentials, http://molmod.ugent.be/deltacodesdft (2015).
[4] K. Lejaeghere, G. Bihlmayer, T. Björkman, P. Blaha, S. Blügel, V. Blum, D. Caliste, I. Castelli, S. Clark, A. D. Corso, S. de Gironcoli, T. Deutsch, J. Dewhurst, I. D. Marco, C. Draxl, M. Duak, O. Eriksson, J. Flores-Livas, K. Garrity, L. Genovese, P. Giannozzi, M. Giantomassi, S. Goedecker, X. Gonze, O. Grånäs, E. Gross, A. Gulans, F. Gygi, D. Hamann, P. Hasnip, N. Holzwarth, D. Iuşan, D. Jochym, F. Jollet, D. Jones, G. Kresse, 
K. Koepernik, E. Küçükbenli, Y. Kvashnin, I. Locht, S. Lubeck, M. Marsman, N. Marzari, U. Nitzsche, L. Nordström, T. Ozaki, L. Paulatto, C. Pickard, W. Poelmans, M. Probert, K. Refson, M. Richter, G.-M. Rignanese, S. Saha, M. Scheffler, M. Schlipf, K. Schwarz, S. Sharma, F. Tavazza, P. Thunström, A. Tkatchenko, M. Torrent, D. Vanderbilt, M. van Setten, V. V. Speybroeck, J. Wills, J. Yates, G.-X. Zhang, and S. Cottenier, Science 351, $\operatorname{aad} 3000$ (2016).

[5] G. van der Laan, J. Phys. Condens. Matter 10, 3239 (1998).

[6] A. A. Khajetoorians and J. Wiebe, Science 344, 976 (2014).

[7] O. Šipr, S. Mankovsky, S. Polesya, S. Bornemann, J. Minár, and H. Ebert, Phys. Rev. B 93, 174409 (2016).

[8] H. J. F. Jansen, G. S. Schneider, and H. Y. Wang, in Electronic Structure and Magnetism of Complex Materials, edited by J. Singh and D. A. Papaconstantopoulos, Springer Series in Materials Science Vol. 54 (Springer, Berlin, 2003), p. 57.

[9] T. Burkert, O. Eriksson, S. I. Simak, A. V. Ruban, B. Sanyal, L. Nordström, and J. M. Wills, Phys. Rev. B 71, 134411 (2005).

[10] P. Błoński and J. Hafner, J. Phys. Condens. Matter 21, 426001 (2009).

[11] J. G. Gay and R. Richter, Phys. Rev. Lett. 56, 2728 (1986).

[12] P. Ravindran, A. Kjekshus, H. Fjellvåg, P. James, L. Nordström, B. Johansson, and O. Eriksson, Phys. Rev. B 63, 144409 (2001).

[13] H. J. F. Jansen, G. S. Schneider, and H. Y. Wang, in Electronic Structure and Magnetism of Complex Materials, edited by D. J. Singh and D. A. Papaconstantopoulos (Springer, Berlin, 2003), p. 57.

[14] O. Šipr, S. Bornemann, J. Minár, and H. Ebert, Phys. Rev. B 82, 174414 (2010).

[15] O. Šipr, S. Bornemann, H. Ebert, and J. Minár, J. Phys. Condens. Matter 26, 196002 (2014).

[16] J. Hu, J. Chen, and G. Ju, in Developments in Data Storage (John Wiley and Sons, Inc., Hoboken, 2011), pp. 223-255.

[17] C. Antoniak, M. E. Gruner, M. Spasova, A. V. Trunova, F. M. Römer, A. Warland, B. Krumme, K. Fauth, S. Sun, P. Entel, M. Farle, and H. Wende, Nat. Commun. 2, 528 (2011).

[18] G. H. O. Daalderop, P. J. Kelly, and M. F. H. Schuurmans, Phys. Rev. B 44, 12054 (1991).

[19] A. Sakuma, J. Phys. Soc. 63, 3053 (1994).

[20] I. V. Solovyev, P. H. Dederichs, and I. Mertig, Phys. Rev. B 52, 13419 (1995).

[21] P. Oppeneer, J. Magn. Magn. Mater 188, 275 (1998).

[22] I. Galanakis, M. Alouani, and H. Dreyssé, Phys. Rev. B 62, 6475 (2000).

[23] A. B. Shick and O. N. Mryasov, Phys. Rev. B 67, 172407 (2003).

[24] S. Ostanin, S. A. Razee, J. B. Staunton, B. Ginatempo, and E. Bruno, J. Appl. Phys. 93, 453 (2003).

[25] T. Kosugi, T. Miyake, and S. Ishibashi, J. Phys. Soc. Jpn. 83, 044707 (2014).

[26] O. A. Ivanov, L. V. Solina, V. A. Demshira, and L. M. Magat, Phys. Met. Metallogr. 35, 92 (1973).

[27] U. von Barth and L. Hedin, J. Phys. C 5, 1629 (1972).

[28] P. Blaha, K. Schwarz, G. K. H. Madsen, D. Kvasnicka, and J. Luitz, Wien2k, An Augmented Plane Wave plus Local orbital Program for Calculating the Crystal Properties (2001), http://www.wien2k.at.

[29] H. Ebert, D. Ködderitzsch, and Minár, Rep. Prog. Phys. 74, 096501 (2011).

[30] H. Ebert, The Munich SPR-KKR package, version 7 (2012), http://olymp.cup.uni-muenchen.de.
[31] O. N. Mryasov, U. Nowak, K. Y. Guslienko, and R. W. Chantrell, Europhys. Lett. 69, 805 (2005).

[32] J. B. Staunton, S. Ostanin, S. S. A. Razee, B. Gyorffy, L. Szunyogh, B. Ginatempo, and E. Bruno, J. Phys. Condens. Matter 16, S5623 (2004).

[33] O. Hovorka, S. Devos, Q. Coopman, W. J. Fan, C. J. Aas, R. F. L. Evans, X. Chen, G. Ju, and R. W. Chantrell, Appl. Phys. Lett. 101, 052406 (2012).

[34] P. V. Lukashev, N. Horrell, and R. F. Sabirianov, J. Appl. Phys. 111, 07A318 (2012).

[35] R. Cuadrado, T. J. Klemmer, and R. W. Chantrell, Appl. Phys. Lett. 105, 152406 (2014).

[36] S. H. Vosko, L. Wilk, and M. Nusair, Can. J. Phys. 58, 1200 (1980).

[37] See Supplemental Material at http://link.aps.org/supplemental/ 10.1103/PhysRevB.94.144436 for more details on the convergence tests.

[38] P. E. Blöchl, O. Jepsen, and O. K. Andersen, Phys. Rev. B 49, 16223 (1994)

[39] T. Huhne and H. Ebert, Phys. Rev. B 65, 205125 (2002).

[40] H. Ebert, H. Freyer, A. Vernes, and G.-Y. Guo, Phys. Rev. B 53, 7721 (1996).

[41] A. H. MacDonald and S. H. Vosko, J. Phys. C 12, 2977 (1979).

[42] J. Kuneš, P. Novák, R. Schmid, P. Blaha, and K. Schwarz, Phys. Rev. B 64, 153102 (2001).

[43] R. Zeller, J. Phys. Condens. Matter 25, 105505 (2013).

[44] A. Alam, S. N. Khan, A. V. Smirnov, D. M. Nicholson, and D. D. Johnson, Phys. Rev. B 90, 205102 (2014).

[45] A. Alam, B. G. Wilson, and D. D. Johnson, Phys. Rev. B 84, 205106 (2011).

[46] R. Kovačik and R. Zeller (private communication).

[47] M. Ogura, C. Zecha, M. Offenberger, H. Ebert, and H. Akai, J. Phys. Condens. Matter 27, 485201 (2015).

[48] A. Alam, S. N. Khan, B. G. Wilson, and D. D. Johnson, Phys. Rev. B 84, 045105 (2011).

[49] R. Zeller, J. Phys. Condens. Matter 27, 306301 (2015).

[50] A. R. Mackintosh and O. K. Andersen, The electronic structure of transition metals, in Electrons at the Fermi Surface, edited by M. Springford (Cambridge University Press, Cambridge, 1980), p. 149.

[51] X. Wang, D. sheng Wang, R. Wu, and A. Freeman, J. Magn. Magn. Mater. 159, 337 (1996).

[52] X. Wang, R. Wu, D.-S. Wang, and A. J. Freeman, Phys. Rev. B 54, 61 (1996).

[53] P. Bruno, Phys. Rev. B 39, 865 (1989).

[54] F. Gimbert and L. Calmels, Phys. Rev. B 86, 184407 (2012).

[55] S. Assa Aravindh, S. Mathi Jaya, M. C. Valsakumar, and C. S. Sundar, Appl. Nanosci. 2, 409 (2012).

[56] Y. Miura, S. Ozaki, Y. Kuwahara, M. Tsujikawa, K. Abe, and M. Shirai, J. Phys. Condens. Matter 25, 106005 (2013).

[57] M. Kotsugi, M. Mizuguchi, S. Sekiya, M. Mizumaki, T. Kojima, T. Nakamura, H. Osawa, K. Kodama, T. Ohtsuki, T. Ohkochi, K. Takanashi, and Y. Watanabe, J. Magn. Magn. Mater. 326, 235 (2013).

[58] T. Ueno, J. Sinha, N. Inami, Y. Takeichi, S. Mitani, K. Ono, and M. Hayashi, Sci. Rep. 5, 14858 (2015).

[59] C. Andersson, B. Sanyal, O. Eriksson, L. Nordström, O. Karis, D. Arvanitis, T. Konishi, E. Holub Krappe, and J. H. Dunn, Phys. Rev. Lett. 99, 177207 (2007).

[60] Y. Kota and A. Sakuma, J. Phys. Soc. 81, 084705 (2012). 
[61] J. W. Davenport, R. E. Watson, and M. Weinert, Phys. Rev. B 37, 9985 (1988).

[62] G. Autès, C. Barreteau, D. Spanjaard, and M.-C. Desjonquères, J. Phys. Condens. Matter 18, 6785 (2006).

[63] J. P. Perdew and Y. Wang, Phys. Rev. B 45, 13244 (1992).

[64] V. L. Moruzzi, J. F. Janak, and A. R. Williams, Calculated Electronic Properties of Metals (Pergamon, New York, 1978).

[65] P. Pyykko and J. P. Desclaux, Acc. Chem. Res. 12, 276 (1979).

[66] K. Nakamura, R. Shimabukuro, Y. Fujiwara, T. Akiyama, T. Ito, and A. J. Freeman, Phys. Rev. Lett. 102, 187201 (2009).

[67] D. Li, A. Smogunov, C. Barreteau, F. Ducastelle, and D. Spanjaard, Phys. Rev. B 88, 214413 (2013).
[68] P. Błoński, A. Lehnert, S. Dennler, S. Rusponi, M. Etzkorn, G. Moulas, P. Bencok, P. Gambardella, H. Brune, and J. Hafner, Phys. Rev. B 81, 104426 (2010).

[69] J. P. Perdew, K. Burke, and M. Ernzerhof, Phys. Rev. Lett 77, 3865 (1996).

[70] M. Brooks, Physica B+C 130, 6 (1985).

[71] O. Eriksson, B. Johansson, R. C. Albers, A. M. Boring, and M. S. S. Brooks, Phys. Rev. B 42, 2707 (1990).

[72] O. Hjortstam, J. Trygg, J. M. Wills, B. Johansson, and O. Eriksson, Phys. Rev. B 53, 9204 (1996).

[73] O. Šipr, J. Minár, S. Mankovsky, and H. Ebert, Phys. Rev. B 78, 144403 (2008). 